\title{
Searches for leptoquarks and heavy leptons with the ATLAS detector at the LHC
}

\section{Sergio Grancagnolo* on behalf of the ATLAS Collaboration}

Humboldt-Universität zu Berlin

E-mail: sergio.grancagnolodcern.ch

\begin{abstract}
Searches of vector-like leptons, heavy Majorana neutrinos, and leptoquarks, using the complete $20.3 \mathrm{fb}^{-1}$ luminosity sample of $p p$ collisions collected at $8 \mathrm{TeV}$ by the ATLAS detector, are presented in this review. Signatures are based on the number of high-momentum leptons, jets, $b$-tagged jets, and on the missing momentum size. No excess was observed in any channel, and limits are set on cross sections, masses and couplings, extending the coverage from previous studies on the considered models.
\end{abstract}

The European Physical Society Conference on High Energy Physics 22-29 July 2015

Vienna, Austria

${ }^{*}$ Speaker. 


\section{Introduction}

In this overview we focus on most recent results on searches for vector-like leptons, heavy Majorana neutrinos, and leptoquarks obtained using the full integrated luminosity $\left(20.3 \mathrm{fb}^{-1}\right)$ of $p p$ collision data collected at $\sqrt{s}=8 \mathrm{TeV}$ by the ATLAS detector [1] at the CERN Large Hadron Collider [2]. In all the analyses being presented, no excess above the expected background from SM events, and no evidence of new heavy particles is observed. Exclusion limits are set at $95 \%$ confidence level (CL) for various masses and mixing parameters, derived from a fit to the considered channels. All systematic uncertainties are supposed to be uncorrelated, and included in the test statistic as nuisance parameters.

\section{Search for vector-like leptons}

Vector-like leptons (VLL) are colourless, spin-1/2 charged fermions and arise in (e.g.) composite Higgs [3] and warped extra dimensions [4] models. In $q \bar{q}$ collisions they are pair-produced in DrellYan processes (Fig. 1, left) [5]. They decay through mixing with electrons or muons ( $\ell$ ) to $W v, Z \ell$, and $H \ell$, with a branching fraction approaching 50\%, 25\%, 25\% respectively, when $m_{\mathrm{L}^{ \pm}} \gg m_{\mathrm{H}}[6]$, while for lower masses the $W v$ mode is favoured. Previous searches performed at LEP, using the $L^{ \pm} \rightarrow W^{ \pm} v$ channel, excluded masses below $m_{\mathrm{L}^{ \pm}}=101.2 \mathrm{GeV}$ [7].

This new ATLAS study assumes at least one heavy lepton to decay into a $Z$ boson, leading to a high-mass resonance decaying to three high transverse momentum $\left(p_{\mathrm{T}}\right)$ leptons. Observed

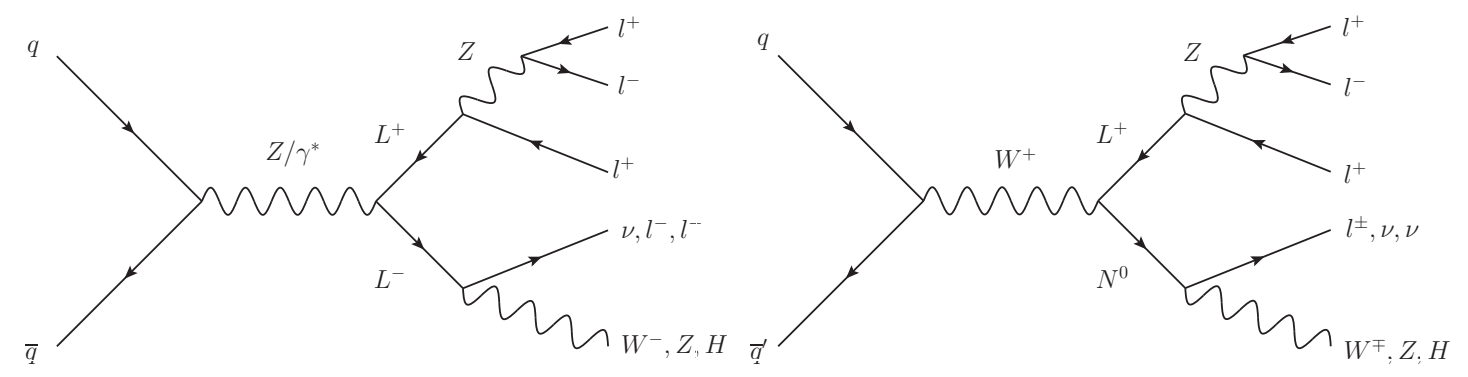

Figure 1: Feynman diagrams for production and decay of new heavy leptons.

(expected) limits on the VLL mass are set for $109 \leq m_{\mathrm{VLL}} \leq 152 \mathrm{GeV}$ and $163 \leq m_{\mathrm{VLL}} \leq 176 \mathrm{GeV}$ $\left(109 \leq m_{\mathrm{VLL}} \leq 152 \mathrm{GeV}\right)$ in the $Z+e$ channel and for $114 \leq m_{\mathrm{VLL}} \leq 153 \mathrm{GeV}$ and $160 \leq m_{\mathrm{VLL}} \leq$ $168 \mathrm{GeV}\left(105 \leq m_{\mathrm{VLL}} \leq 167 \mathrm{GeV}\right)$ in the $Z+\mu$ channel (Fig. 2) [8].

\section{Type-III seesaw mechanism}

New SU(2) triplets with zero hypercharge can be introduced with a Type-III seesaw mechanism, that generate neutrino masses and couple to gauge bosons [9]. Charged and neutral heavy leptons are possible, the first behaving as in VLL (Fig. 1, left), while the neutral $\left(N^{0}\right)$ decaying to $W \ell, Z v$, and $H v$ (Fig. 1, right). Since in case of a mass splitting the decay within the heavy leptons are highly suppressed, their three masses are assumed to be degenerate. Previous searches from CMS 

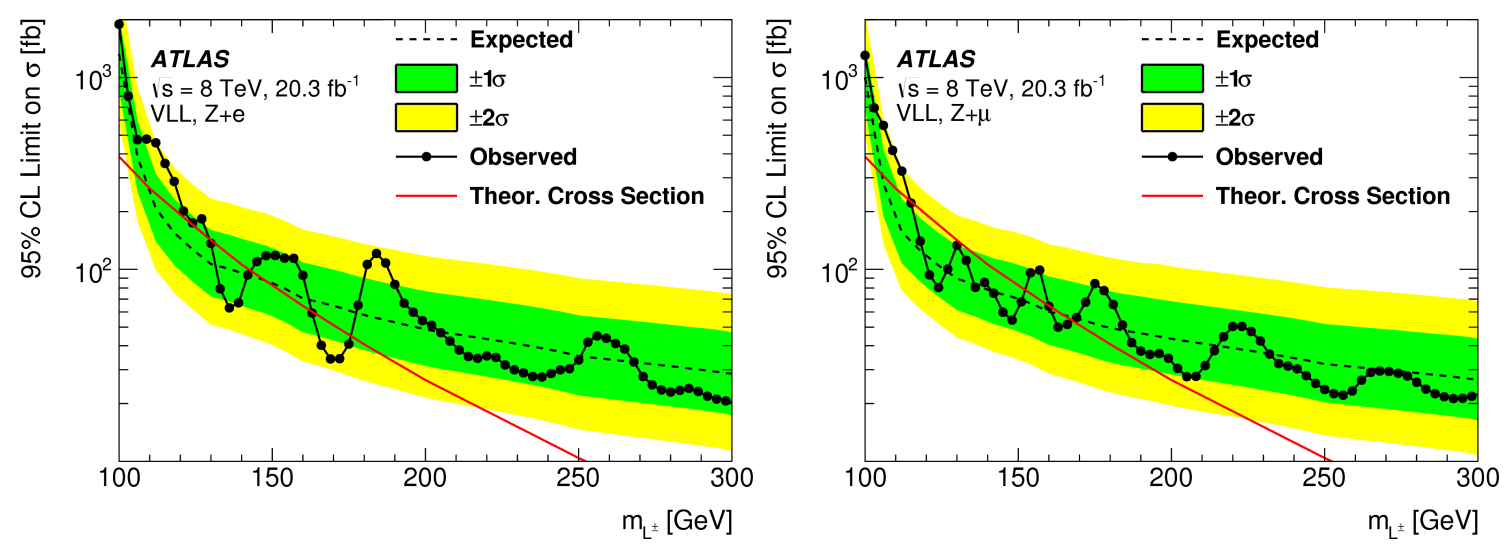

Figure 2: Pair-production cross section [6] and VLL mass limits. Left (right): $Z+e(Z+\mu)$ channel [8].

excluded Type-III seesaw fermions with masses below $m_{\mathrm{L}^{ \pm}}<180-210 \mathrm{GeV}$, depending on the considered scenario [10].

Two new searches were performed by ATLAS. In the first, requiring at least three leptons, observed (expected) limits are set on the Type-III seesaw generated heavy-lepton mass for $100 \leq$ $m_{\mathrm{L}^{ \pm}} \leq 430 \mathrm{GeV}\left(100 \leq m_{\mathrm{L}^{ \pm}} \leq 436 \mathrm{GeV}\right)$ in the $Z+e$ channel and for $100 \leq m_{\mathrm{L}^{ \pm}} \leq 401 \mathrm{GeV}$ and $419 \leq m_{\mathrm{L}^{ \pm}} \leq 468 \mathrm{GeV}\left(100 \leq m_{\mathrm{L}^{ \pm}} \leq 419 \mathrm{GeV}\right)$ in the $Z+\mu$ channel (Fig. 3) [8].
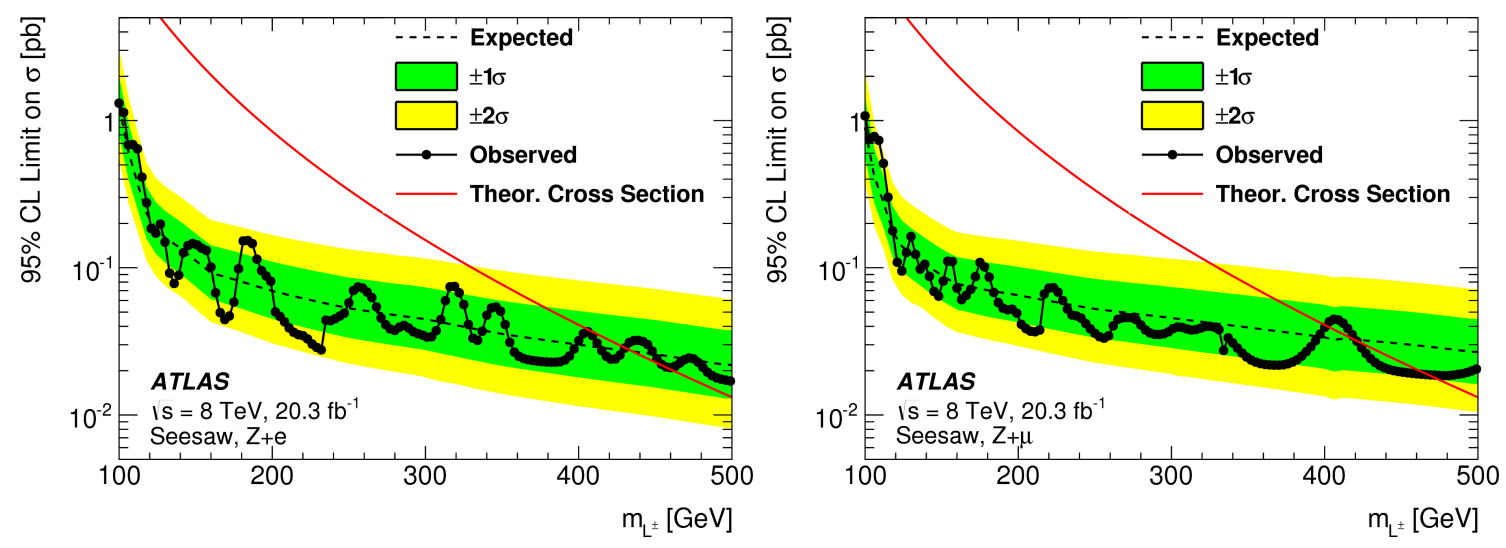

Figure 3: Limits on the Type-III seesaw heavy-lepton mass. Left (right): $Z+e(Z+\mu)$ channel [8].

The second search looks for the process $p p \rightarrow N^{0} L^{ \pm} \rightarrow W^{ \pm} \ell^{\mp} W^{ \pm} v$. The final state requires two leptons ( $e$ or $\mu$ ) of any charge, at least two jets, of which those with the highest $p_{\mathrm{T}}$ are required to have an invariant mass consistent with the $W$-boson mass, and large missing transverse momentum $\left(E_{\mathrm{T}}^{\mathrm{miss}}\right)$. Examining a benchmark point with no coupling with $\tau$ lepton $\left(V_{\tau}=0\right)$, observed limits are set for different mixing hypotheses: $m_{\mathrm{L} / \mathrm{N}} \leq 325 \mathrm{GeV}$ for $V_{\mu}=0, m_{\mathrm{L} / \mathrm{N}} \leq 400 \mathrm{GeV}$ for $V_{e}=0$, $m_{\mathrm{L} / \mathrm{N}} \leq 335 \mathrm{GeV}$ for $V_{e} / V_{\mu}=0.87$, and $m_{\mathrm{L} / \mathrm{N}} \leq 475 \mathrm{GeV}$ assuming $\mathrm{BR}\left(L, N^{0} \rightarrow W v, \ell\right)=100 \%$ (Fig. 4) [11].

\section{Majorana neutrinos}

Two theoretical extensions of the SM containing Majorana neutrinos are considered here. The 

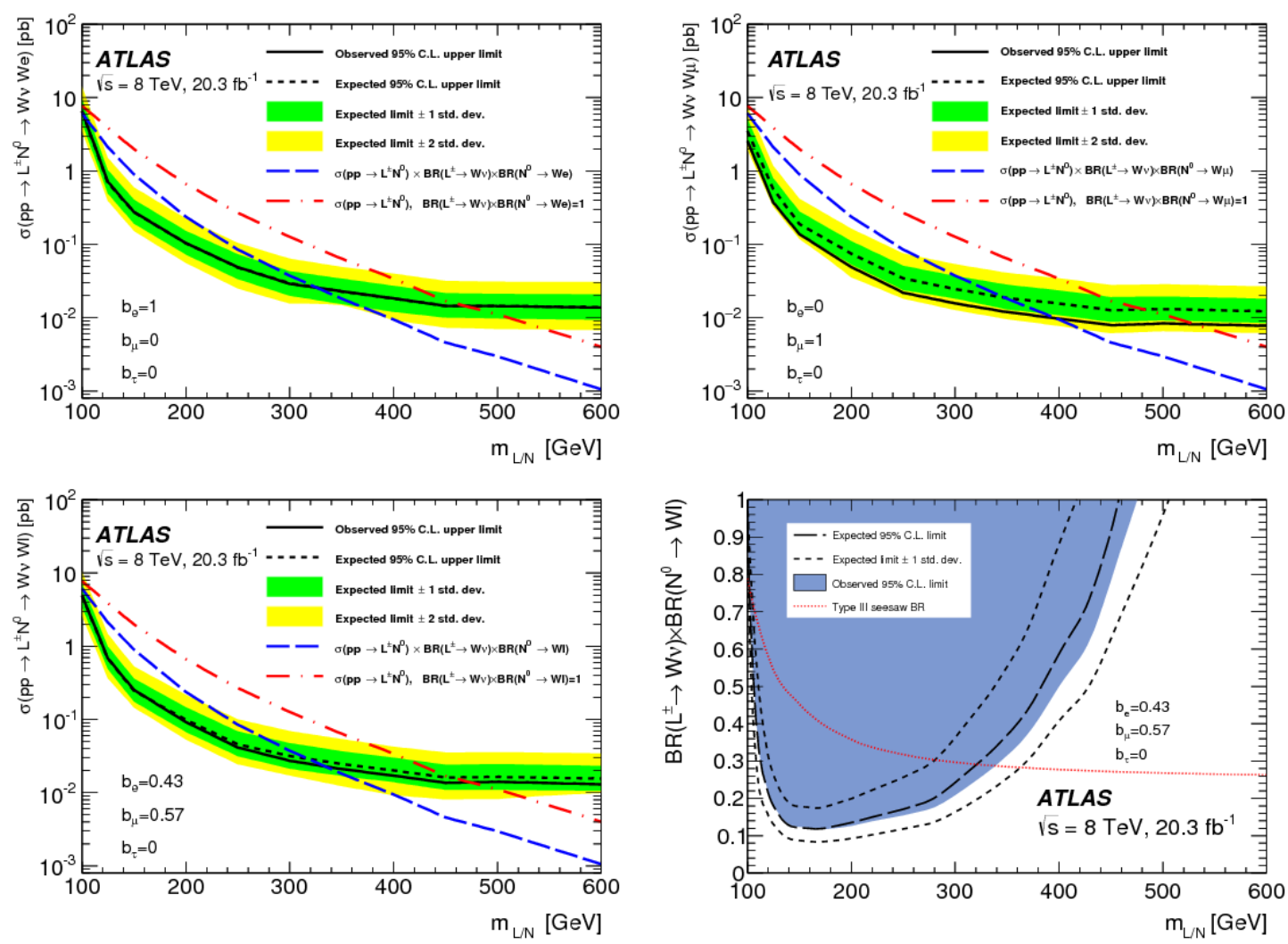

Figure 4: Limits on the Type-III seesaw heavy-lepton mass for the dilepton + dijet $+E_{\mathrm{T}}^{\text {miss }}$ channel for different mixing hypotheses. Top-left (top-right): exclusive coupling to electrons (muons). Bottom-left: $V_{e} / V_{\mu}=0.87$. Bottom-right: upper limits on $\mathrm{BR}\left(L^{ \pm} \rightarrow W v\right) \times \mathrm{BR}\left(N \rightarrow W^{ \pm} \ell^{\mp}\right)$ versus $m_{\mathrm{L} / \mathrm{N}}[11]$.

first model include right-handed neutrinos, in a minimal Type-I seesaw light and heavy $v$ mass generation (mTISM) [12]. Heavy Majorana neutrinos $(N)$ can be produced via an off-shell W boson and then decay on-shell to $W \ell$ (Fig. 5, left). In the second model, a right-handed symmetry $S U(2)_{\mathrm{R}}$ analogue of SM $S U(2)_{\mathrm{L}}$ is added (LRSM), predicting gauge bosons $W_{\mathrm{R}}, Z^{\prime}$ assumed to be more massive than heavy neutrinos [13]. Those can be produced through $W_{\mathrm{R}} \rightarrow N \ell, Z^{\prime} \rightarrow N N$ and then decay via $N \rightarrow \ell W_{\mathrm{R}}^{*}\left(\rightarrow q \bar{q}^{\prime}\right)$ (Fig. 5, right).

In previous searches, LEP excluded mTISM heavy neutrino masses $m_{\mathrm{N}}<m_{Z}[14,15]$, and CMS excluded $90<m_{\mathrm{N}}<200 \mathrm{GeV}$ (ee channel), $40<m_{\mathrm{N}}<500 \mathrm{GeV}$ ( $\mu \mu$ channel) [16, 17]; ATLAS excluded LRSM heavy gauge bosons with mass $m_{W_{R}}<2.3 \mathrm{TeV}$, for $m_{W_{R}}-m_{\mathrm{N}}>0.3 \mathrm{TeV}$ [18], and CMS excluded $m_{W_{R}}<3.0 \mathrm{TeV}$, for $m_{W_{R}}-m_{\mathrm{N}}>0.05 \mathrm{TeV}$ [19].

New ATLAS searches are performed using exactly two leptons (ee or $\mu \mu$ ) of the same charge, and optimizing the event selection for each of the two models. Since the mTISM final state assumes the $W$-boson decaying into $q \bar{q}$, it is required to have a small $E_{\mathrm{T}}^{\text {miss }}$ and at least two jets (those with the highest $p_{\mathrm{T}}$ having an invariant mass compatible with the $W$ mass). The LRSM $W_{\mathrm{R}}\left(Z^{\prime}\right)$ selection asks for at least one or two (two or four) jet(s) and a large invariant mass of the lepton pair, and of the leptons plus jet(s) system. Limits are set on the production cross section as a function of the $m_{\mathrm{N}}$ (Fig. 6), and on the mixing matrix elements with SM neutrinos (Fig. 7), as low as $\left|V_{e N}\right|^{2}=0.029$ 

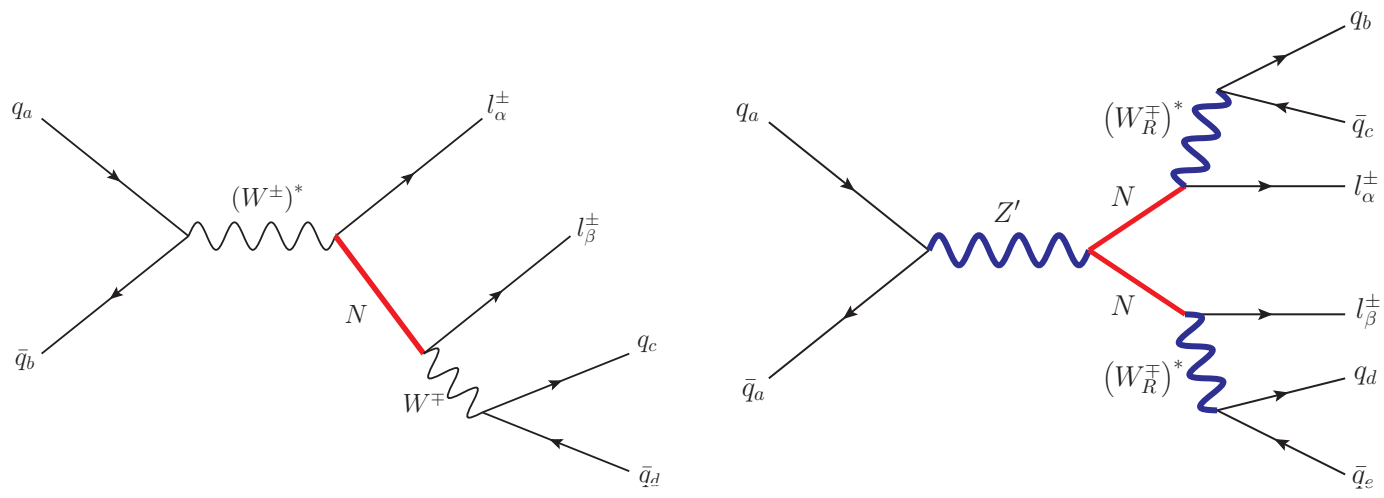

Figure 5: Feynman diagrams for production and decay of new heavy neutrinos.
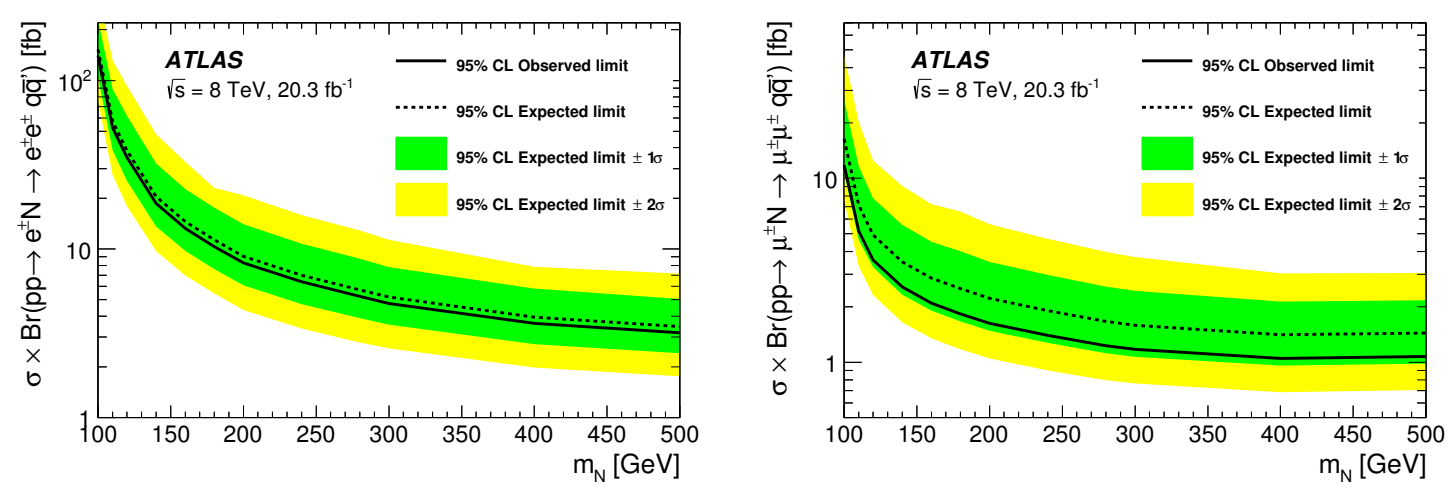

Figure 6: Limits on the production cross section of Type-I seesaw heavy Majorana neutrinos as a function of mass. Left (right): dielectron (dimuon) plus jets channel. [20].
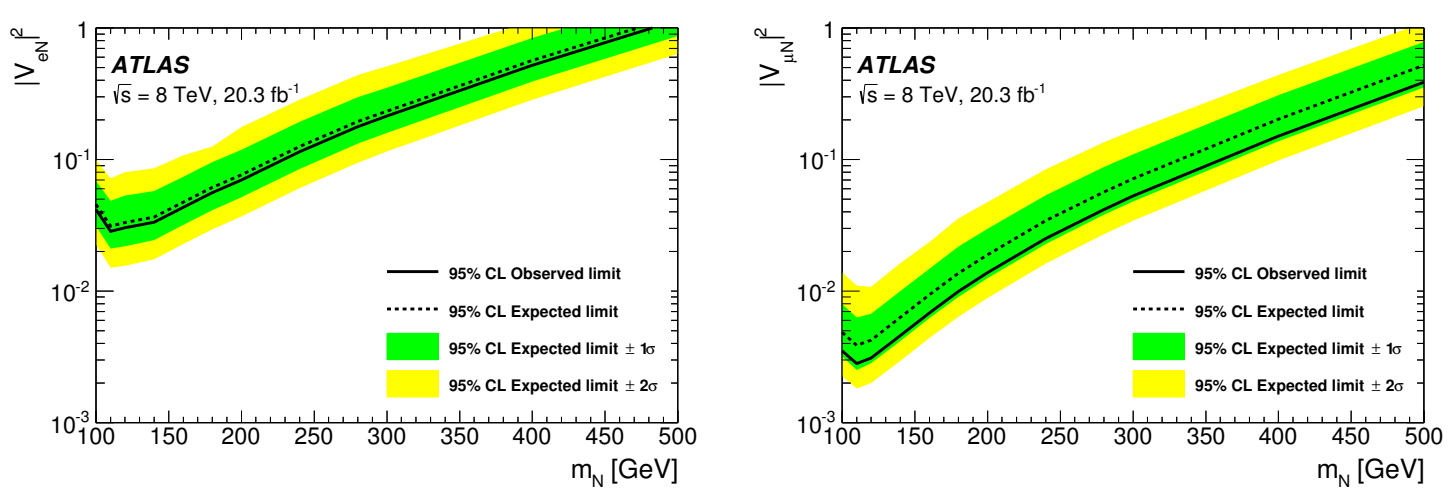

Figure 7: Left (right): limits on the mixing of Type-I seesaw heavy Majorana neutrinos with $e(\mu)[20]$. 
and $\left|V_{\mu N}\right|^{2}=0.0028$ at $m_{\mathrm{N}} \approx 110 \mathrm{GeV}$. Exclusion contours are also set in the $m_{W_{\mathrm{R}}}-m_{\mathrm{N}}$ and $m_{Z^{\prime}}-m_{\mathrm{N}}$ plane (Fig. 8) [20].
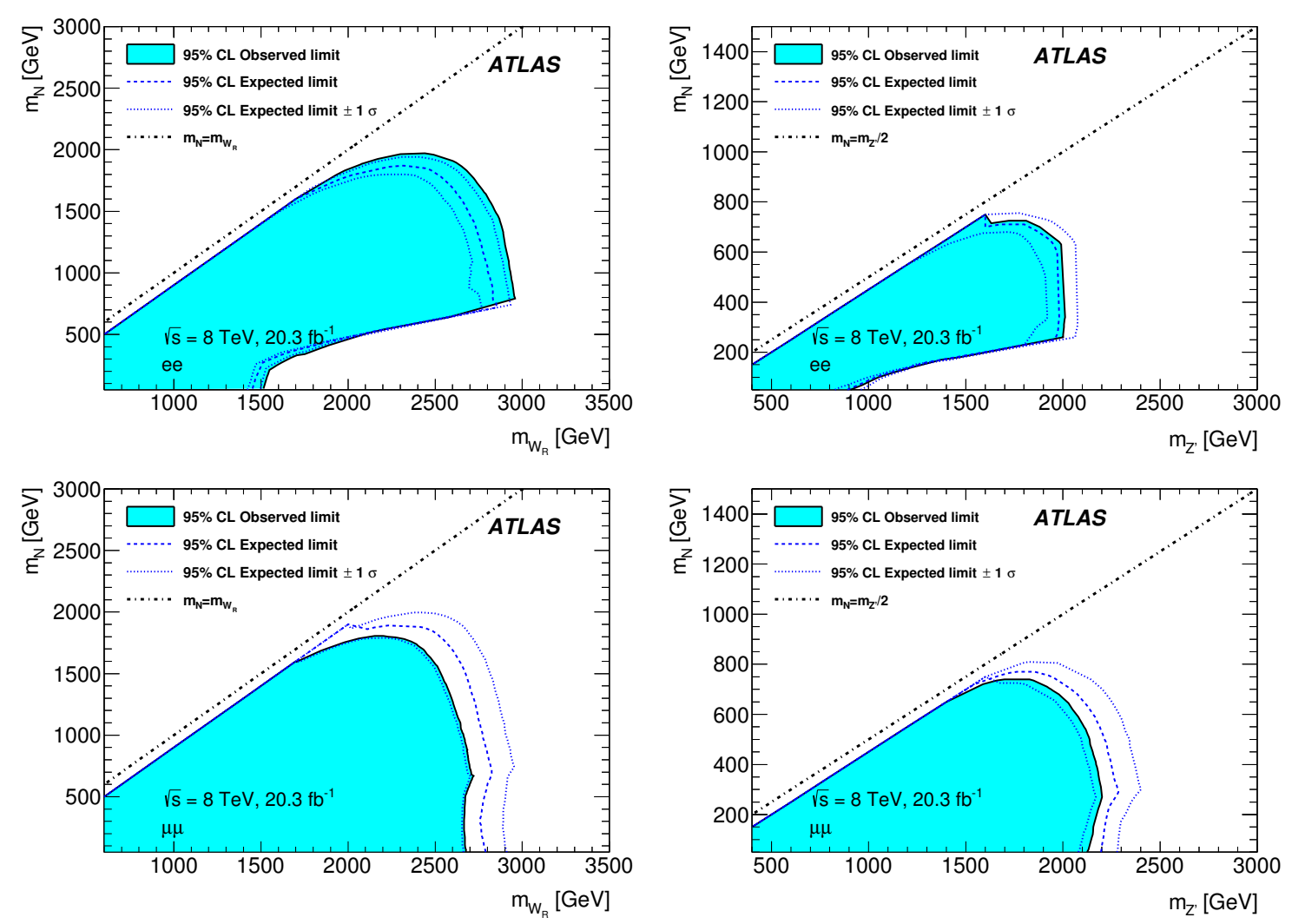

Figure 8: Left (right): limits on the LRSM heavy gauge bosons $W_{\mathrm{R}}\left(Z^{\prime}\right)$. Top (bottom): dielectron (dimuon) channel. [20].

\section{Leptoquarks}

Leptoquarks (LQs) are colour triplet bosons with fractional electric charge, carrying both nonzero baryon and lepton numbers. The dominant pair-production mechanism is $g g$ fusion for $m_{\mathrm{LQ}}<$ $1 \mathrm{TeV}$ and $q q$-annihilation at higher LQ masses. For providing more conservative limits, only scalar production is taken in account. The branching fraction $\beta$ of the decay into a charged lepton, and the Yukawa coupling $\lambda_{L Q \rightarrow l q}$ that determines lifetime, are model dependent. In the model considered here, LQs are grouped into three generations, each decaying only into a $l q$ pair of the corresponding SM family [21].

Previous searches from ATLAS and CMS set the following limits: $m_{\mathrm{LQ} 1}<830(640) \mathrm{GeV}$ at $\beta=1(0.5), m_{\mathrm{LQ} 2}<840(650) \mathrm{GeV}$ at $\beta=1(0.5), m_{\mathrm{LQ} 3}<440 \mathrm{GeV}$ in the channel $L Q_{3} \rightarrow b v_{\tau}$, $m_{\mathrm{LQ} 3}<740 \mathrm{GeV}$ for $L Q_{3} \rightarrow b \tau, m_{\mathrm{LQ} 3}<685 \mathrm{GeV}$ for $L Q_{3} \rightarrow t \tau$ [22-29].

ATLAS performed a new search for all three generation scalar LQs. The search for $1^{\text {st }}$ and $2^{\text {nd }}$ generation LQs used final states with at least two jets and either two electrons of any charge or two muons with opposite charge. Various signal regions are defined, based on the minimum value of 
the dilepton invariant mass $\left(m_{\ell \ell}\right)$, the scalar sum of transverse momenta of the leptons and the two leading jets $\left(S_{\mathrm{T}}\right)$, and the lowest reconstructed LQ mass in the event $\left(m_{\mathrm{LQ}}^{\min }\right)$. They were optimised in a three-dimensional space of those variables, targeting different ranges of LQ masses (Table 1). The distribution of the $m_{\mathrm{LQ}}^{\min }$ variable after applying pre-selection cuts is shown in Fig. 9. The cross

\begin{tabular}{c|c|ccc}
\hline & $\begin{array}{c}\text { LQ masses } \\
{[\mathrm{GeV}]}\end{array}$ & $\begin{array}{c}m_{\ell \ell} \\
{[\mathrm{GeV}]}\end{array}$ & $\begin{array}{c}S_{\mathrm{T}} \\
{[\mathrm{GeV}]}\end{array}$ & $\begin{array}{c}m_{\mathrm{LQ}}^{\min } \\
{[\mathrm{GeV}]}\end{array}$ \\
\hline SR1 & 300 & 130 & 460 & 210 \\
SR2 & 350 & 160 & 550 & 250 \\
SR3 & 400 & 160 & 590 & 280 \\
SR4 & 450 & 160 & 670 & 370 \\
SR5 & $500-550$ & 180 & 760 & 410 \\
SR6 & $600-650$ & 180 & 850 & 490 \\
SR7 & $700-750$ & 180 & 950 & 580 \\
SR8 & $800-1300$ & 180 & 1190 & 610 \\
\hline
\end{tabular}

Table 1: Minimum values of $m_{\ell \ell}, S_{\mathrm{T}}$, and $m_{\mathrm{LQ}}^{\min }$ used to define each of the signal regions targeting different leptoquark masses in the $e e j j$ and $\mu \mu j j$ channels.
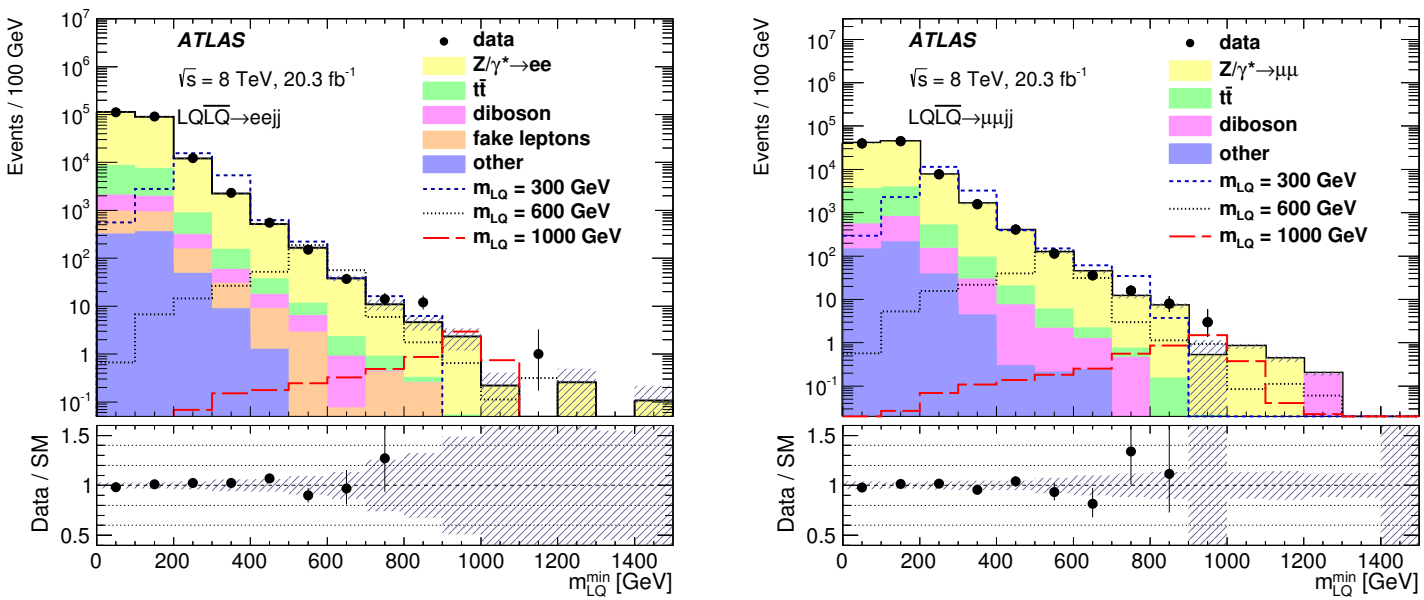

Figure 9: Left (right): reconstructed LQs invariant mass distribution after pre-selection cuts for the $e e j j$ $(\mu \mu j j)$ channel [30].

section limits for $\beta=1$ are presented in Fig. 10 and as a function of $\beta$ in Fig. 11. First (second) generation scalar LQs are excluded for $m_{\mathrm{LQ} 1}<1050 \mathrm{GeV}\left(m_{\mathrm{LQ} 2}<1000 \mathrm{GeV}\right)$ at $\beta=1$ [30].

ATLAS reinterpreted two searches for third generation supersymmetric partners of bottom and top quarks [31,32] in terms of a third-generation LQ (LQ3) decaying to a $b$-quark and a $v_{\tau}$ and of a LQ3 going into a $t$-quark and a $v_{\tau}(\beta=0)$. In the first case, events are required to have exactly two $b$-tagged jets and large $E_{\mathrm{T}}^{\text {miss }}$. In the second case, events are required to have one lepton, jets, and $E_{\mathrm{T}}^{\text {miss }}$. The observed (expected) exclusion mass limits are $m_{\mathrm{LQ} 3}<625 \mathrm{GeV}\left(m_{\mathrm{LQ} 3}<640 \mathrm{GeV}\right)$ in the $b v_{\tau} \bar{b} \bar{v}_{\tau}$ channel and $210<m_{\mathrm{LQ} 3}<640 \mathrm{GeV}\left(200<m_{\mathrm{LQ} 3}<685 \mathrm{GeV}\right)$ in the $t v_{\tau} \bar{\tau} \bar{v}_{\tau}$ channel (Fig. 12) [30]. 

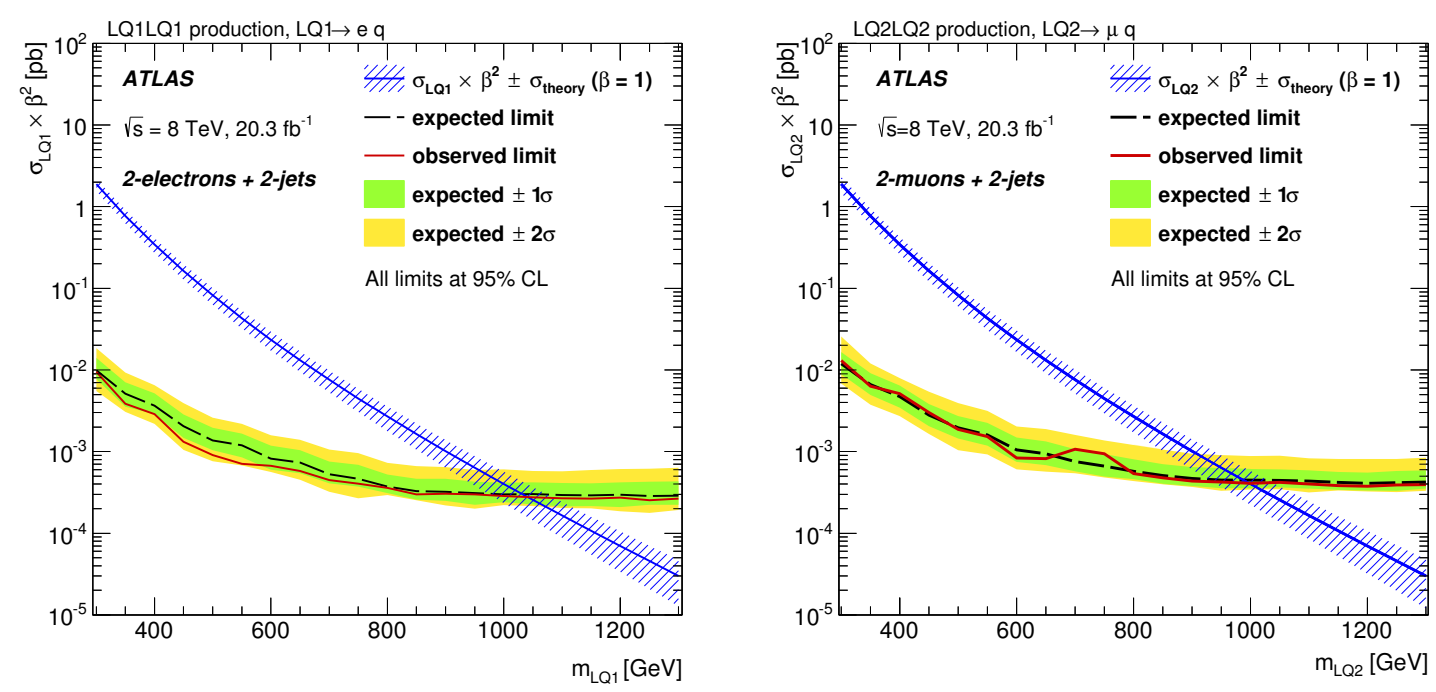

Figure 10: Left (right): Cross section limits as a function of LQ1 (LQ2) mass for the eejj $(\mu \mu j j)$ channel [30].
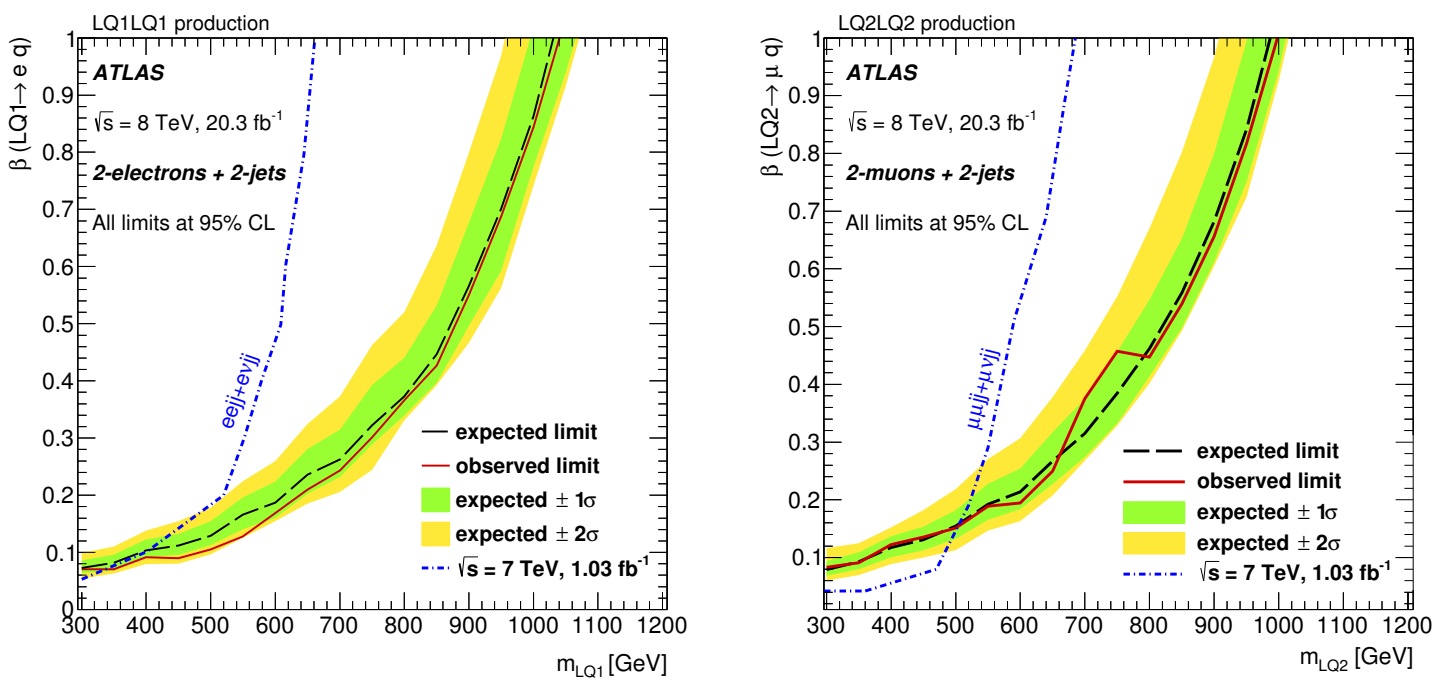

Figure 11: Excluded LQ1 (LQ2) mass as a function of the branching ratio $\beta$. Left (right): eejj $(\mu \mu j j$ ) channel [30]. The blue dashed-dotted lines show the former $7 \mathrm{TeV}$ limits from ATLAS [29].

\section{Conclusions}

By exploiting the complete $20.3 \mathrm{fb}^{-1}$ luminosity of $p p$ collisions collected at $8 \mathrm{TeV}$, ATLAS has tested various models predicting heavy leptons, Majorana neutrinos, and leptoquarks. Different finales states were explored, based on the number of high-momentum leptons, jets, and $b$-tagged jets. Missing momentum and other kinematic variables were used to define signal and background control regions. No excess was observed in any channel, and limits are set on cross sections, masses and couplings, extending the coverage from previous researches. 

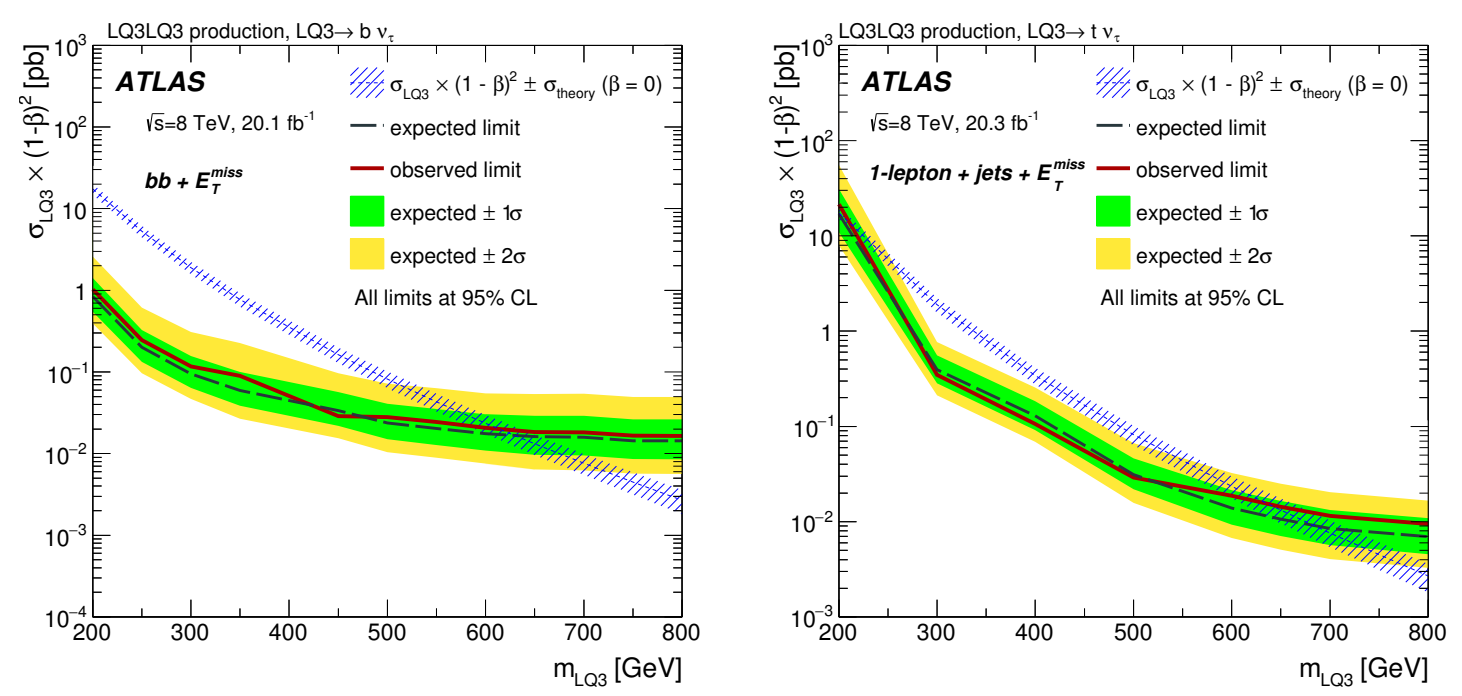

Figure 12: Left (right): LQ3 pair production limits in the channel $b v_{\tau} \bar{b} \bar{v}_{\tau}\left(t v_{\tau} \bar{t} \bar{v}_{\tau}\right)$ [30].

\section{References}

[1] ATLAS Collaboration, The ATLAS Experiment at the CERN Large Hadron Collider, JINST 3 (2008) S08003.

[2] L. Evans and P. Bryant, Lhc machine, Journal of Instrumentation 3 (2008), no. 08 S08001.

[3] K. Fujikawa, A Vector-like extension of the standard model, Prog. Theor. Phys. 92 (1994) 1149-1160, [hep-ph/9411258].

[4] R. Contino, T. Kramer, M. Son, and R. Sundrum, Warped/composite phenomenology simplified, JHEP 05 (2007) 074, [hep-ph/ 0612180$].$

[5] A. Falkowski, D. M. Straub, and A. Vicente, Vector-like leptons: Higgs decays and collider phenomenology, JHEP 05 (2014) 092, [arXiv: 1312. 5329].

[6] S. P. Martin, Extra vector-like matter and the lightest Higgs scalar boson mass in low-energy supersymmetry, Phys. Rev. D81 (2010) 035004, [arXiv: 0910.2732].

[7] L3 Collaboration, P. Achard et al., Search for heavy neutral and charged leptons in $e^{+} e^{-}$annihilation at LEP, Phys. Lett. B517 (2001) 75-85, [hep-ex/0107015].

[8] ATLAS Collaboration, Search for heavy lepton resonances decaying to a $Z$ boson and a lepton in $p p$ collisions at $\sqrt{s}=8 \mathrm{TeV}$ with the ATLAS detector, JHEP 09 (2015) 108, [arXiv:1506.1291].

[9] C. Biggio and F. Bonnet, Implementation of the Type III Seesaw Model in FeynRules/MadGraph and Prospects for Discovery with Early LHC Data, Eur. Phys. J. C72 (2012) 1899, [arXiv:1107.3463].

[10] CMS Collaboration, Search for heavy lepton partners of neutrinos in proton-proton collisions in the context of the type $\{$ III $\}$ seesaw mechanism, Phys. Lett. B 718 (2012) 348 - 368.

[11] ATLAS Collaboration, Search for type-III Seesaw heavy leptons in pp collisions at $\sqrt{s}=8 \mathrm{TeV}$ with the ATLAS Detector, Phys. Rev. D92 (2015) 032001, [arXiv: 1506.1839 ]. 
[12] A. Atre, T. Han, S. Pascoli, and B. Zhang, The Search for Heavy Majorana Neutrinos, JHEP 05 (2009) 030, [arXiv:0 901.3589 ].

[13] R. N. Mohapatra and G. Senjanović, Neutrino mass and spontaneous parity nonconservation, Phys. Rev. Lett. 44 (Apr, 1980) 912-915.

[14] DELPHI Collaboration, P. Abreu et al., Search for neutral heavy leptons produced in $Z$ decays, $Z$. Phys. C74 (1997) 57-71. [Erratum: Z.Phys.C75,580(1997)].

[15] L3 Collaboration, O. A. et al, Search for isosinglet neutral heavy leptons in zO decays, Phys. Lett. B 295 (1992) $371-382$.

[16] CMS Collaboration, Search for heavy majorana neutrinos in and events in pp collisions at $\sqrt{s}=7 \mathrm{TeV}$, Phys. Lett. B 717 (2012) $109-128$.

[17] CMS Collaboration, Search for heavy Majorana neutrinos in $\mu^{ \pm} \mu^{ \pm}+$jets events in proton-proton collisions at $\sqrt{s}=8$ TeV, Phys. Lett. B748 (2015) 144-166, [arXiv: 1501.5566 ].

[18] ATLAS Collaboration, Search for heavy neutrinos and right-handed $W$ bosons in events with two leptons and jets in pp collisions at $\sqrt{s}=7 \mathrm{TeV}$ with the ATLAS detector, Eur. Phys. J. C72 (2012) 2056, [arXiv:1203.5420].

[19] CMS Collaboration, Search for heavy neutrinos and $\mathrm{W}$ bosons with right-handed couplings in proton-proton collisions at $\sqrt{s}=8 \mathrm{TeV}$, Eur. Phys. J. C74 (2014) 3149, [arXiv: 1407.3683 ].

[20] ATLAS Collaboration, Search for heavy Majorana neutrinos with the ATLAS detector in pp collisions at $\sqrt{s}=8 \mathrm{TeV}$, JHEP 07 (2015) 162, [arXiv:1506.6020].

[21] W. Buchmüller, R. Rückl, and D. Wyler, Leptoquarks in lepton-quark collisions, Phys. Lett. B 191 (1987) $442-448$.

[22] CMS Collaboration, Search for pair production of first- and second-generation scalar leptoquarks in pp collisions at $\sqrt{s}=7 \mathrm{TeV}$, Phys. Rev. D86 (2012) 052013, [arXiv: 1207.5406 ].

[23] CMS Collaboration, Search for Pair-production of First Generation Scalar Leptoquarks in pp Collisions at sqrt $s=8 \mathrm{TeV}$, CMS-PAS-EXO-12-041 (2014).

[24] ATLAS Collaboration, Search for first generation scalar leptoquarks in pp collisions at $\sqrt{s}=7 \mathrm{TeV}$ with the ATLAS detector, Phys. Lett. B709 (2012) 158-176, [arXiv: 1112 . 4828]. [Erratum: Phys. Lett.B711,442(2012)].

[25] ATLAS Collaboration, Search for second generation scalar leptoquarks in pp collisions at $\sqrt{s}=7$ TeV with the ATLAS detector, Eur. Phys. J. C72 (2012) 2151, [arXiv: 1203.3172].

[26] CMS Collaboration, Search for third-generation leptoquarks and scalar bottom quarks in pp collisions at $\sqrt{s}=7 \mathrm{TeV}, \mathrm{JHEP} 12$ (2012) 055, [arXiv: 1210 . 5627].

[27] CMS Collaboration, Search for pair production of third-generation scalar leptoquarks and top squarks in proton-proton collisions at sqrt(s) $=8 \mathrm{TeV}$, Phys. Lett. B739 (2014) 229, [arXiv:1408.0806].

[28] CMS Collaboration, Search for Third-Generation Scalar Leptoquarks in the t $\tau$ Channel in Proton-Proton Collisions at $\sqrt{s}=8 \mathrm{TeV}, \mathrm{JHEP} 07$ (2015) 042, [arXiv: 1503.9049 ].

[29] ATLAS Collaboration, Search for third generation scalar leptoquarks in pp collisions at $\sqrt{s}=7 \mathrm{TeV}$ with the ATLAS detector, JHEP 06 (2013) 033, [arXiv: 1303.0526 ].

[30] ATLAS Collaboration, Searches for scalar leptoquarks in pp collisions at $\sqrt{s}=8 \mathrm{TeV}$ with the ATLAS detector, arXiv:1508.4735. 
[31] ATLAS Collaboration, Search for direct third-generation squark pair production in final states with missing transverse momentum and two b-jets in $\sqrt{s}=8 \mathrm{TeV}$ pp collisions with the ATLAS detector, JHEP 10 (2013) 189, [arXiv:1308.2631].

[32] ATLAS Collaboration, Search for top squark pair production in final states with one isolated lepton, jets, and missing transverse momentum in $\sqrt{s}=8 \mathrm{TeV}$ pp collisions with the ATLAS detector, JHEP 11 (2014) 118, [arXiv:1407.0583]. 